Check for updates

Cite this: Nanoscale Adv., 2019, 1, 781

\title{
A technique-driven materials categorisation scheme to support regulatory identification of nanomaterials $\uparrow$
}

\author{
Claire Gaillard, $\S^{\mathrm{a}}$ Agnieszka Mech, (DD ${ }^{\mathrm{a}}$ Wendel Wohlleben, ${ }^{\mathrm{b}}$ Frank Babick, (D) ${ }^{\mathrm{c}}$ \\ Vasile-Dan Hodoroaba, ${ }^{d}$ Antoine Ghanem, ${ }^{e}$ Stefan Weigel $\left(\mathbb{D} t^{f}\right.$ \\ and Hubert Rauscher (iD *a
}

Worldwide there is a variety of regulatory provisions addressing nanomaterials. The identification as nanomaterial in a regulatory context often has the consequence that specific legal rules apply. In identifying nanomaterials, and to find out whether nanomaterial-specific provisions apply, the external size of particles is globally used as a criterion. For legal certainty, its assessment for regulatory purposes should be based on measurements and methods that are robust, fit for the purpose and ready to be accepted by different stakeholders and authorities. This should help to assure the safety of nanomaterials and at the same time facilitate their international trading. Therefore, we propose a categorisation scheme which is driven by the capabilities of common characterisation techniques for particle size measurement. Categorising materials according to this scheme takes into account the particle properties that are most important for a determination of their size. The categorisation is exemplified for the specific particle number based size metric of the European Commission's recommendation on the definition of nanomaterial, but it is applicable to other metrics as well. Matching the performance profiles of the measurement techniques with the material property profiles (i) allows selecting the most appropriate size determination technique for every type of material considered, (ii) enables proper identification of nanomaterials, and (iii) has the potential to be accepted by regulators, industry and consumers alike. Having such a scheme in place would facilitate the regulatory assessment of nanomaterials in regional legislation as well as in international relations between different regulatory regions assuring the safe trade of nanomaterials.

Received 27th August 2018 Accepted 1st November 2018

DOI: $10.1039 / c 8 n a 00175 h$

rsc.li/nanoscale-advances

\section{The need for a technique-driven materials categorisation scheme}

The increasing presence of nanomaterials (NMs) in commercial products has triggered the need for their regulation and development of specific methodology to demonstrate that their use is

\footnotetext{
${ }^{a}$ European Commission, Joint Research Centre, 21027 Ispra (VA), Italy. E-mail: hubert. rauscher@ec.europa.eu

${ }^{b}$ BASF SE, Material Physics Research, 67056 Ludwigshafen, Germany

'Technische Universität Dresden, Institute of Process Engineering and Environmental Technology, Dresden, Germany

${ }^{d}$ Federal Institute for Materials Research and Testing (BAM), 12205 Berlin, Germany ${ }^{e}$ Solvay - Research \& Innovation Centre Brussels, 1120 Brussels, Belgium

${ }^{{ }_{R} R I K I L T}$ - Wageningen UR, 6708 Wageningen, The Netherlands

$\dagger$ Electronic supplementary information (ESI) available. See DOI: 10.1039/c8na00175h

$\S$ Present address: Université de Lyon, Université Claude Bernard Lyon 1, MATEIS CNRS UMR 5510, Faculté de Pharmacie - ISPB, 8 avenue Rockefeller, 69373 Lyon Cedex 08, France.

\$ Present address: German Federal Institute for Risk Assessment (BfR), 10589 Berlin, Germany.
}

safe. Consequently, worldwide a variety of legislative provisions in different sectors address nanomaterials and require their identification, characterisation, quantification, and often a specific safety assessment. In the $\mathrm{EU}$, the regulations on medical devices, ${ }^{1}$ biocidal products, ${ }^{2}$ novel foods ${ }^{3}$ and cosmetic products ${ }^{4}$ specifically address nanomaterials and we can expect the same for the European chemicals regulation (REACH) once its amended annexes, which are currently under revision, are adopted. The United States Food and Drug Administration (US FDA) has issued guidance on considering whether an FDAregulated product involves the application of nanotechnology and the United States Environmental Protection Agency (US EPA) has just recently published rules for reporting and recordkeeping of certain chemical substances when they are manufactured or processed at the nanoscale. ${ }^{6}$ Nanomaterials are subject to specific regulatory scrutiny in other countries as well. ${ }^{7}$ Commercial firms (e.g. producer, supplier) and authorities have to comply with such provisions and should have the same understanding of the regulatory requirements. This holds true within regional regulation as well as in the trade relations between regions with different regulations. 
Specific regulatory provisions include definitions of the term 'nanomaterial' to identify a material as nano- or nonnanomaterial according to certain criteria, and to decide if nanomaterial-specific provisions apply. In the European Commission's recommendation on the definition of 'nanomaterial', size is the only relevant property, ${ }^{8}$ whereas in other regulatory contexts additional characteristics, such as origin, biopersistence or novel nanospecific physicochemical properties, are relevant as well. ${ }^{9}$ Regardless of differences in scope and implementation, all definitions of the term 'nanomaterial' share one common feature as the fundamental defining element: the particle size. Consequently, in any context for a decision whether a material is a nanomaterial or not, it is always necessary to determine its particle size distribution. This involves the measurement of particle size from few nanometres up to well into the micrometre range (around $10 \mu \mathrm{m}$ )..$^{10}$ Although particle size can be determined by a large variety of analytical techniques, each technique has its region of applicability in terms of material classes, material properties and the accessible size range, including the medium in which the particles are dispersed. None of the available techniques is suitable for all materials. ${ }^{11-18}$ However, if such size measurements are to be done to fulfil regulatory obligations, the results must be relevant, reliable and transparent so that the involved parties, i.e. commercial firms and regulators, including non-specialists in the metrology field, mutually can accept the conclusions drawn from them. For this reason, it is essential that such measurements are performed with well-established and reliable methods that are fit for the purpose and that they can be carried out with reasonable economic effort.

To cope with all these challenges, it is necessary to come to an agreement on which techniques can be used for which materials and for which purpose. To select the most appropriate technique(s) one should match material properties with the regions of applicability and the performance profile of size measurement techniques. In the case of particulate materials, the availability of a knowledge base consisting of size measurement techniques matched to specific material properties would greatly facilitate a reliable regulatory valid identification as a nanomaterial or nonnanomaterial.

We distinguish between the terms 'techniques' and 'methods'. A characterisation technique, or measurement technique, is a measurement process that is based on a specific physical principle, e.g. electron microscopy or mass spectrometry. According to the physical principle there are limitations of each technique with view to size and materials that can be analysed (e.g. organic vs. metal). An analysis/ analytical method is the application of a technique to a specific material, eventually in a specific matrix, including all necessary steps of sample preparation and data analysis. Methods are specific to the respective analyte/matrix combination. The short terms 'method' and 'technique' are used in the sense as explained above throughout this article. The categorisation suggested in this paper is addressing techniques, not individual methods.

\section{Categorisation criteria}

Nanomaterials have been categorised in the context of risk assessment, ${ }^{19,20}$ detection in complex media such as foods, ${ }^{17}$ systematic description for nanomaterial data resources ${ }^{\mathbf{2 1}}$ or toxicology. ${ }^{22,23}$ Various schemes to group nanomaterials, depending on their chemical nature, ${ }^{24}$ hazard to human health, ${ }^{25}$ commercial importance ${ }^{26}$ or physiological modes of action, ${ }^{23,27}$ were proposed in the literature. However, what is lacking is a practical system for the fundamental task to select appropriate particle sizing techniques for all kinds of particulate materials. The generated measurement results should then be sufficiently robust to serve as a basis for a decision whether a material meets a regulatory size criterion for nanomaterials or not.

Here we propose a material categorisation scheme (MCS) which is technique-driven and pragmatic to facilitate the regulatory identification of nanomaterials.

Materials are categorised, i.e., divided into groups, according to criteria linked to the capabilities of experimental techniques for particle size measurement. This allows the selection of techniques that are compatible and suited to measure materials with specific characteristics, which in turn assures the reliability and common acceptance of the obtained data. The term 'categorisation' here is used strictly according to the general meaning of the word (putting things into groups that have the same features ${ }^{28}$ ). In this sense the MCS serves as a technical guideline to choose an appropriate technique and remains unrelated to regulatory concepts such as classification of substances for assessing hazards and risks.

\subsection{Material types}

Considering the performance characteristics of specific techniques, i.e. what kind of material they actually can characterise in a reliable way, we propose three classes of materials to start with when categorising a given material (see Fig. 1):

Material with monotype particles. All particles are of the same type, i.e. they have essentially the same chemical and structural composition. For the purposes of specific legislation an ensemble of such particles can constitute a 'nanoform' as defined by the European Chemicals Agency ${ }^{29}$ and a 'discrete form' of a substance in the nanoscale as defined by US $\mathrm{EPA}^{6}$ or non-nanoform under specific conditions. The particles can consist of (i) a single chemical element (e.g. Au) or compound (e.g. $\mathrm{SiO}_{2}$ ) or (ii) different elements or compounds, but with the same internal structure. In the latter case, the particles are composite $^{\mathbf{3 0}}$ particles. Composite particles can be present in different types as well. Core-shell particles consist of at least two components, one of which (the core) lies within the other that forms the outer layer (the shell). Multishell particles are core-shell particles with more than one outer layer (shell). Particles with inclusions are particles in which the components are phase-separated from each other and one phase is dispersed in the other and forms the inclusions. The number and size of the domains can vary, and their spatial distribution within the particles is often not uniform. The internal structure of 


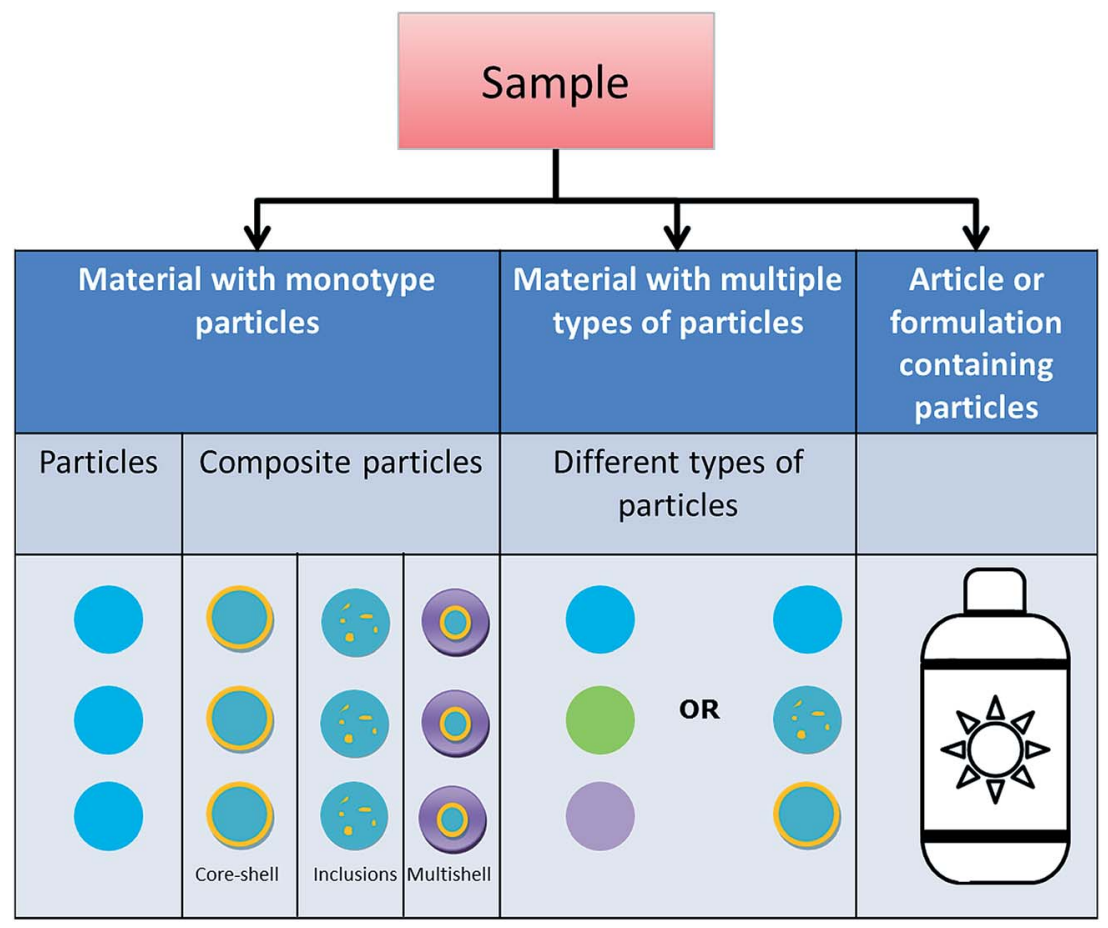

Fig. 1 Different types of particles and particulate materials are considered in the categorisation scheme. 'Sample' is the generic term for the material to be analysed.

a composite particle can be important for characterisation purposes. For example, certain techniques such as spICP-MS (Single Particle Inductively coupled plasma-mass spectrometry) or XRD (X-ray diffraction) cannot accurately measure the size of multilayer particles.

Materials with multiple types of particles. A material that contains particles of different types, i.e. different chemical or structural compositions, can be visualised as a mixture of different materials with monotype particles.

Articles and formulations that contain particles of the same or different types: an article is an object which, during production, is given a special shape, surface or design which determines its function to a greater degree than does its chemical composition. ${ }^{31}$ An article may consist of different chemical substances in different physical phases (liquid/solid/ gaseous) and forms, including nanoparticles of one or several types. A formulation is a particular combination of chemicals (prepared according to a formula) that do not chemically react with each other. The chemicals in a formulation are chosen because of their specific properties, and, when combined, result in a product with desirable characteristics. This also includes certain consumer products, which are defined according to CEN as items intended for consumers or likely to be used by consumers. ${ }^{32}$ For example, a sunscreen that contains titanium dioxide nanoparticles is a formulation and a tennis racquet with incorporated carbon nanotubes is an article. Both of them are consumer products.

If the particles are all of the same type, a characterisation technique needs to be suitable for that type only, whereas if a material consists of different particle types, the chosen technique should be applicable to all particle types present. The choice of the most appropriate technique(s) to measure particle size is further determined by the properties of the material to measure since the latter may determine the techniques' limits of applicability. Hence, only a good match between the material properties and the performance of the technique will lead to reliable and robust results. This holds true for the detection and characterisation of nanoparticles in pure substances ${ }^{11}$ as well as in more complex environments. ${ }^{12-14,16,17}$

\subsection{Selection of categorisation criteria}

After the determination of the material class, the material is further categorised according to the following sub-classes that describe the most relevant particle parameters which dominate the choice of the analytical technique(s) for particle size determination:

Chemical composition. The chemical nature of the particles strongly influences the choice of the appropriate characterisation techniques. ${ }^{33}$ This may be due to a compositiondependent sensitivity, or because some techniques may be applied only to a limited variety of chemicals, e.g. due to element-specific detection. ${ }^{11}$ Taking into account the performance of techniques for particle size measurement, this criterion is further divided into six specific subtypes which allow categorising most of the currently available nanomaterials:

- Inorganic materials (e.g. metals and their alloys, oxides and sulfides, salts, silicates), except carbon.

- Pure carbon-based materials (CNTs, nanodiamonds, carbon black....).

- Organic particulate materials (polymers, pigments, etc.). 
- Biological materials (nucleic acids, peptides, proteins), including synthetic biological materials.

- Other (dendrimers, liposomes, supramolecular assemblies...).

- Materials consisting of composite particles.

- Unknown.

The above-shown division is based on the assumption that in all subtypes, except for composite and unknown particles, the elements are homogeneously distributed across the particles. Consequently, e.g. a core-shell particle that consists of a Ag core and $\mathrm{Au}$ shell cannot be categorised as inorganic even though it consists of inorganic elements, but it is categorised as a composite particle.

Organic particulate materials like polymer lattices or pigments are relatively straightforward to measure due to their constituent particle boundaries, despite potentially challenging aggregate structures and shape issues. On the other hand, the category called 'other' such as dendrimers or supramolecular assemblies can be challenging due to their structure and complex chemical composition. For instance, sizing by Ultrasonic Spectroscopy (USSp), where the diameter (acoustophoretic diameter) is calculated based on spherical particle estimation, cannot measure properly these types of materials.

Biological materials such as nucleic acids or proteins were grouped separately because of their possible sensitivity to some testing procedures. Their spatial conformations are sensitive to $\mathrm{pH}$ and temperature, and a denaturation of their structure and functions due to experimental conditions would render them different in comparison to the original sample.

The chemical nature of the particles strongly influences the choice of the appropriate characterisation techniques. Actually, certain techniques, e.g. spICP-MS $^{\mathbf{3 4}}$ are very sensitive to the elemental composition of the analysed sample and thus the use of spICP-MS for instance is limited to inorganic materials with sufficiently high atomic weight.

Composite particles deserve specific attention. It is therefore necessary to know if a specific technique is able to determine the particle size without interference caused by the individual composite structure.

It is generally advantageous that the user has knowledge on the chemical composition of the material before measurement of the particle size distribution is attempted. If the chemical composition is entirely unknown, it is advisable to do a chemical analysis, otherwise a method suitable for unknown chemical composition must be selected. The same is true if very high contamination is suspected which would affect the outcome of a measurement technique.

Number of small dimensions and shape. Many of the currently employed characterisation methods implicitly assume that the particles are spherical or yield an equivalent spherical size (for example DLS), ${ }^{33}$ which severely limits their applicability to particles with a non-spherical shape. ${ }^{36}$ Furthermore, techniques need to be specifically suitable to measure the smallest dimensions of plate- or fibre-like particles. An additional difficulty occurs if the analysed sample consists of a mixture of particles of different shapes. In such a case, only electron microscopy (EM) and possibly scanning probe microscopy could yield reliable results. Even with EM, the analysis of platelike particles and the accurate evaluation of their thickness distribution are problematic as the smallest dimension (thickness) could be difficult to access.

Considering the characteristics of available analytical techniques, the criteria of shape and number of nanoscale dimensions of nanoparticles can assume the following values:

Number of nanoscale dimensions:

- 1

- 2

- 3

- Mixture of nanoparticles with different shapes.

In principle, indication of the number of small dimensions is sufficient for the purposes of the intended categorisation scheme, but often it is helpful to characterise particle shape with more descriptive terms. They are also included here regardless of some redundancy with the criterion of number of small dimensions. Descriptive criteria for particle shape are:

- One small dimension: plates (flat shapes incl. irregular flakes) (ratio large:small dimensions $\geq 3$ ). $\uparrow$

- Two small dimensions: fibres (elongated shapes such as tubes, fibres, rods) (ratio length:diameter $\geq 3$ ). $\uparrow$

- Three small dimensions: spherical, equiaxial or similar (e.g. prismatic, cubic, tetrahedral).

- Mixture of nanoparticles with different shapes.

- Other (incl. unknown).

Size range. Techniques for particle sizing have their own measurement range. The size range therefore is definitely a criterion which can limit the choice of the techniques to obtain an accurate result. ${ }^{35}$ That size range can depend on further criteria, e.g., the chemical composition or the polydispersity of the material. In practice, the analysis needs to cover the entire size range of the particulate material in order to get an accurate result for the size distribution. ${ }^{36}$ If the particle size range in a sample is too large, certain techniques cannot determine the actual particle size distribution. DLS, for example, is much more sensitive to large particles than to small ones, which can easily lead to inaccurate size distribution results for particulate materials with a broad particle size distribution. Moreover, other techniques such as Tunable Resistive Pulse Sensing (TRPS) are not able to measure very small particles. ${ }^{33}$ In both cases, an overestimation of the measured particle size is the consequence. Conversely, small angle X-ray scattering (SAXS) is not able to measure particles above $100 \mathrm{~nm}$, this making the technique prone to underestimate the median particle size for broad size distributions well above $100 \mathrm{~nm}$; this finally results in possible false-positive results, i.e. identification of a non-nanomaterial as a nanomaterial.

Trade form and dispersibility. Some characterisation techniques require the particles to be dispersed in a liquid phase, whereas others only work for powders. Therefore, it is essential to know if the substance to analyse is pre-dispersed or can be

I According to ISO/TS 80004-2:2015(en), terms such as nanofibre or nanoplate may be preferred to the term nanoparticle if the dimensions differ significantly (typically by more than 3 times). 
dispersed, including information on the dispersing media and specific protocols to be used, in order to determine which characterisation technique could be suitable for the analysis. As recently discussed by Hartmann et al., ${ }^{37}$ particle size distributions can be affected by many factors, such as sample preparation protocols, including the choice of the dispersion media, particle concentration or material tendency to aggregation and agglomeration.

The user should therefore first indicate the trade form of the sample, which can be

- Powder

- Suspension

- Aerosol

Considering the characteristics of the evaluated techniques, materials are categorised further as:

- Dispersible in aqueous media (by generalised protocols).

- Dispersible in material-specific media and protocols.

- Can be aerosolised.

Stability during testing. Some materials may be incompatible with the conditions of certain measurement techniques, e.g., they may be sensitive to irradiation by electrons. If this is the case, they cannot be characterised reliably with EM, or require more sophisticated EM techniques, such as cryo-EM or soft excitation conditions (low beam current or voltage) techniques. Particularly polymers or organic solids may be degraded by electron beam irradiation. ${ }^{38-40}$ Other substances may be stable in a narrow temperature range. ${ }^{41}$ Therefore, it is generally necessary to know if characterisation techniques can induce by their probes or by operating conditions (for example vacuum in EM) damages to sensitive materials.

The criteria addressed here relate to sensitivity against

- Electron beam irradiation

- Vacuum conditions

- Heating (with specification of the maximum acceptable temperature)

- Cooling (with specification of the lowest acceptable temperature)

- If a material releases ions, atoms or molecules in its environment, this can also interfere with measurements, therefore this criterion is also included. For instance, as Ag particles dissolve, nanoparticles can actually remain undetected.

Specific properties. Specific electrical, optical, magnetic and surface properties may interfere with or, in contrast, facilitate certain measurement techniques. ${ }^{36}$ Specific material properties are therefore to be taken into account in order to avoid inappropriate techniques.

For instance, an electrically insulating material cannot be analysed in conventional SEM unless it is coated with a thin layer of conductive material. ${ }^{42}$ The charging effect arising from electron/ion irradiation can be otherwise avoided to a certain extent if the SEM instrument used has a low voltage option or a variable pressure option. ${ }^{43}$

In addition to standard techniques, magnetic particles may also be easily characterised with some particular techniques, e.g. magnetic force microscopy (MFM), in which a sharp magnetised tip maps the magnetic force gradient above the sample surface while simultaneously acquiring topographical data. ${ }^{44}$

Some materials also have specific spectroscopic properties. For instance, Raman spectroscopy can be used to measure the diameter of single walled carbon nanotubes. ${ }^{45} \mathrm{UV}$-Vis spectrometry can also serve as a size measurement technique if the material exhibits Surface Plasmon Resonances, ${ }^{46}$ e.g. silver or gold nanoparticles.

Another criterion is the presence of a functionalisation or coating of the particles, i.e., if the outermost layer has a different chemical nature than the core of the particles. This modifies the characteristics of the particle surface, e.g. the presence of hydrophobic, hydrophilic, reactive groups. Some characterisation techniques - especially those where the measurements are performed in a liquid dispersion - can be affected by these features. For example, the analysis may lead to artefacts especially when the hydrodynamic diameter is measured, and this fact also needs to be taken into consideration for the sample preparation.

Functionalisation is distinguished in this MCS from the coating which is defined as a uniform layer. For instance, a particle completely covered by a dense layer will belong to the composite category. On the other hand, a particle with molecules bound to the surface in a less dense layer, where the surface of the particle is still accessible by other molecules, is considered here as a functionalised particle. Characterisation techniques such as DLS (measuring hydrodynamic diameter) or USSp (measuring acoustophoretic diameter) ${ }^{33}$ will measure the whole ensemble and will give a false estimation of the particle size.

For the reasons described above, it can be important to be aware of the following specific properties of the sample:

- Electrical conductivity, i.e., whether the material is conductive, semi-conductive or insulating.

- Magnetic properties.

- Size-dependent absorption of photons/fluorescence. If yes, which technique could be used for characterisation, e.g. UV or fluorescence spectrometry.

- Functionalisation, coating and chemical composition of the surface layer.

- Other specific surface properties of the particles.

We can further sub-divide each of these main criteria to specify in detail material characteristics that are relevant for particle sizing techniques. The resulting proposed MCS is presented in Fig. 2 for materials with monotype particles. Not only can the proposed MCS serve as a powerful tool supporting regulatory identification of nanomaterials but it should also be helpful for academia, industry and other stakeholders when choosing the most appropriate technique for development, research or quality control (QC) purposes involving particulate materials, including nanomaterials.

For illustration of the categorisation system, let us consider a hypothetical material that consists of monotype particles. That material can either directly match one of the sub-criteria (e.g. main criterion: chemical composition $\rightarrow$ sub-criterion: carbon based) or have a value associated with a sub-criterion 


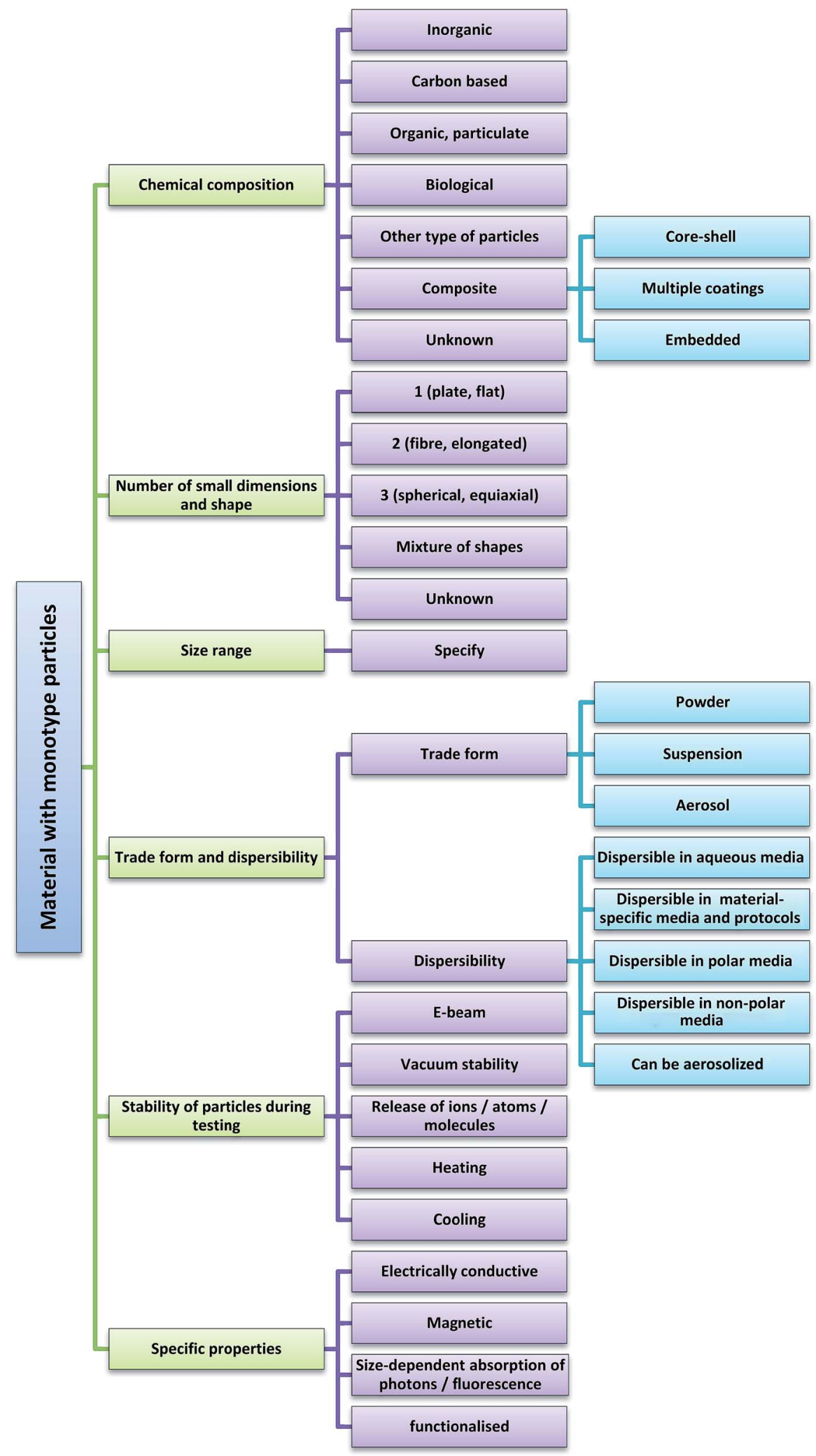

Fig. 2 Overview of the material categorisation system for a material with monotype particles.

(e.g. stability of particles during testing $\rightarrow$ heating $\rightarrow$ stable up to ...K). A material fully categorised this way can then be matched to the performance of available particle size measurement techniques, which finally allows the selection of the appropriate and most suitable techniques for a given material. 


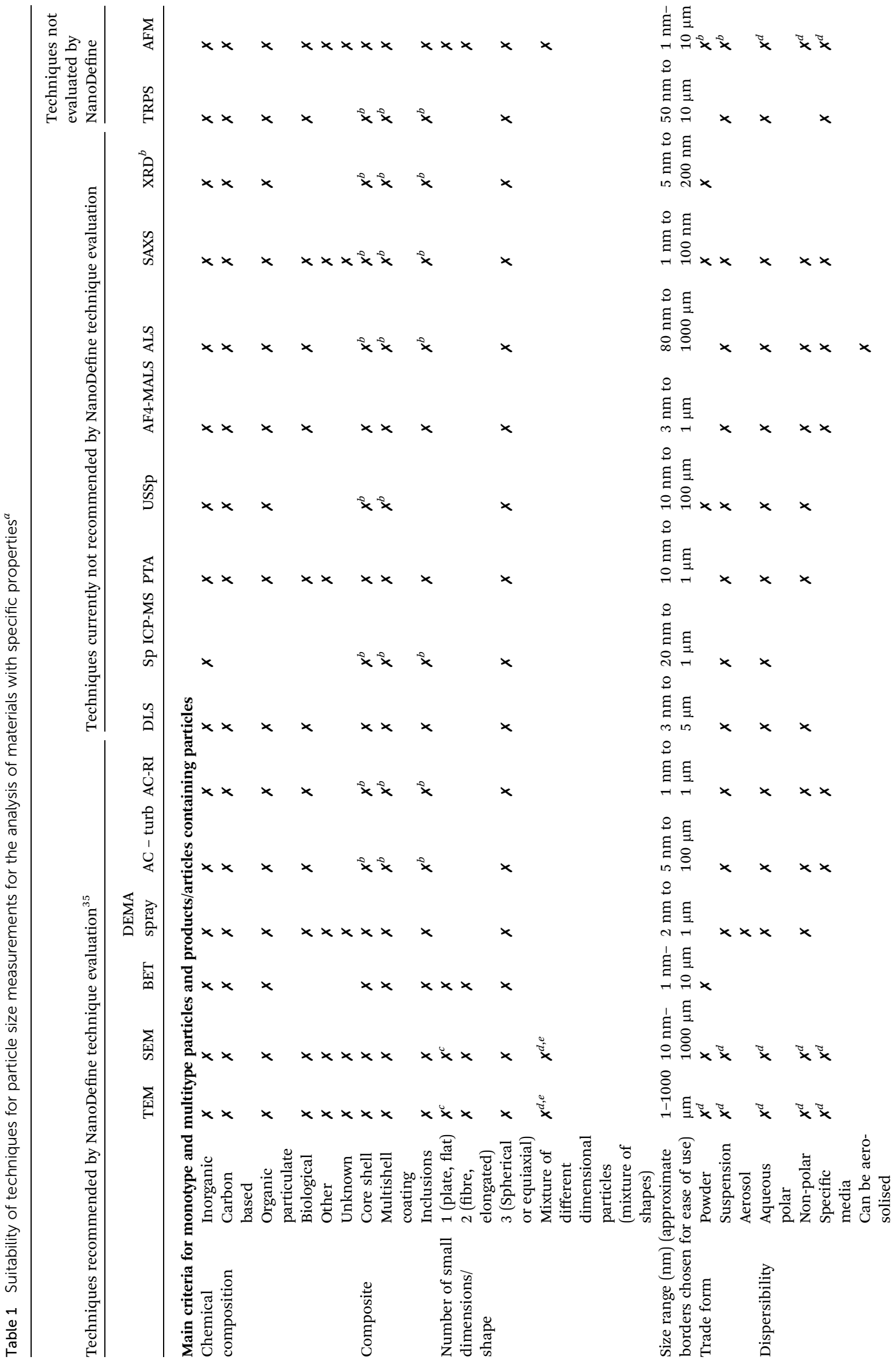




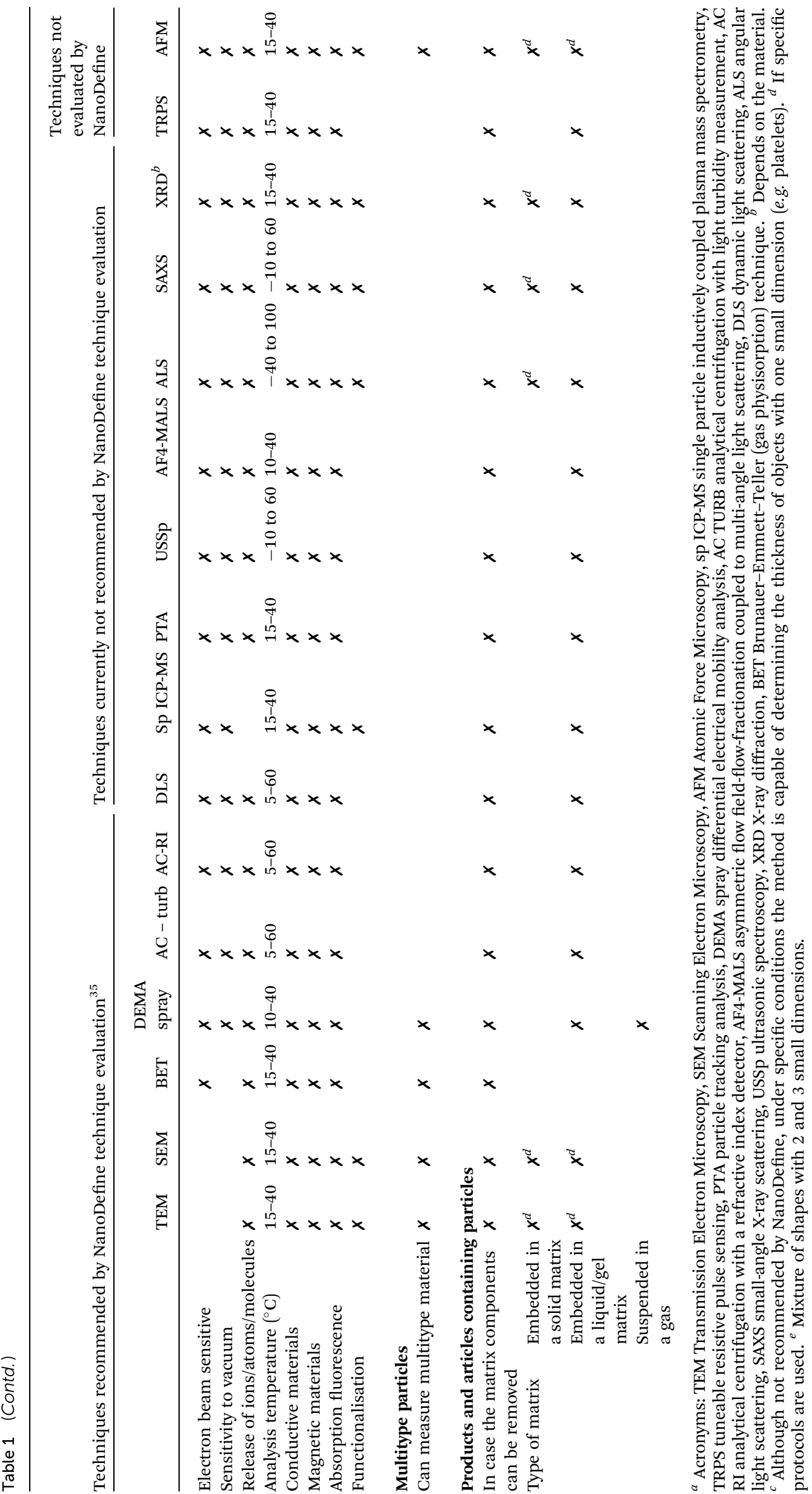


The MCS can also be applied to materials with multiple particle types, i.e. where particles do not have the same chemical composition and internal structure, by applying the categorisation to each individual type of particles of the material. Ideally, the analysis of such a material employs selective techniques to measure the size distribution of each particle type independently of the other type(s). This is typically possible only if one deals with a mixture of different substances or a mixture of different types of nanoparticles with non-overlapping size distributions.

Similarly, categorisation of particles in an article or a formulation is also possible (see the ESI, $\dagger$ with an example). In that case, criteria for the possibility to remove the nonparticulate matrix (defined here as a non-particulate constituent or component of a material, including additives) are added while other criteria remain the same. Addressing also particulate materials in the MCS which are incorporated in articles or products aims to facilitate the identification of the particles for regulatory enforcement when required.

\section{Matching material properties and experimental capabilities}

The performance of a broad range of widely available and frequently used techniques to determine particle size was systematically evaluated in the NanoDefine ${ }^{47}$ EU project against the criteria of the MCS, ${ }^{33}$ using a set of representative examples, including well-defined quality control materials as well as industrial materials of complex shapes and considerable polydispersity. This way, specific regions of applicability of the individual techniques in terms of materials categorisation criteria, e.g. material classes, chemical composition, size range, trade forms etc. ${ }^{35}$ were established.

The resulting categorisation matrix can be used to match materials with known specific properties according to the MCS to the techniques best suitable to analyse particle size. Table 1 shows the results of this evaluation.

This table is also integrated as a configuration file in the NanoDefiner e-tool, ${ }^{48}$ a free software to support the identification of nanomaterials according to the EC NM definition. As the performance of nanoparticle sizing techniques may be improved and new measurement techniques may become available in the future, the table can be updated and complemented according to the latest developments and thus be kept up to date. Guidelines issued by authorities usually list and describe techniques recommended for regulatory purposes.

The MCS proposed here and the mapping of the technique performance criteria (see Table 1) could be included in such guidance. Applying them to cases where a regulatory decision on the identification of a material as a nanomaterial (with all its regulatory consequences) is necessary would certainly help to harmonise nanomaterial identification.

However, a final decision whether a material meets the size criteria for a nanomaterial as defined under a specific regulatory rule not only requires selecting the appropriate technique(s) for a specific material. It also needs considerations on the measurement uncertainty associated with the result obtained with a specific method in combination with a particular material. If a material is far away from the borderline separating nano- from non-nanomaterials, a larger measurement uncertainty can be accepted as compared to when it is a borderline material and identification as nano- or non-nanomaterial is more difficult. The latter would require in-depth confirmatory methods to come to a reliable identification. Such different levels of complexity in the analysis could be taken into account in a tiered method approach as discussed by Babick et al. ${ }^{35}$

In addition to selecting suitable techniques for the measurement of particle size distributions by applying the MCS, samples need to be appropriately prepared for the measurements, which is usually material and technique-specific. ${ }^{37,49}$ Guidance for application of sizing techniques should therefore include or refer to available information on sample preparation, i.e., material and technique specific preparation protocols, if available, or to general guidance on sample preparation. Such integrated guidance is also currently in preparation. ${ }^{47}$

\section{An example: gold nanorods}

In the following, we present a simple example where the MCS is applied to a material consisting of monotype particles. Another, more complex, example for categorisation of a material where the particles are embedded in a matrix can be found in the ESI. $\dagger$

In the example selected here, the material consists of a suspension of gold nanorods. The task is to identify the most appropriate characterisation technique(s) that would allow determining whether this is a nanomaterial according to the EC recommendation.

We assume that the following information on the material is available according to the categorisation scheme. The chemical composition is 'inorganic' (gold), two dimensions are expected to be smaller than the third dimension (hence the shape is 'elongated'), the size range of the smallest dimension is expected to be between ' $40 \mathrm{~nm}$ ' and ' $90 \mathrm{~nm}$ ' and the trade form is 'suspension'. The gold nanorods are 'dispersible in aqueous media', 'stable under electron beam irradiation' and 'in vacuum'. A 'release of molecules, atoms or ions' is not expected. The particles are stable at least 'between $-100{ }^{\circ} \mathrm{C}$ and $400{ }^{\circ} \mathrm{C}$ '. They are 'electrically conductive' and have 'unknown magnetic properties'. There may be a 'size-dependent absorption of photons' and the surface is 'not functionalised'.

Matching these material properties with the technique's performance characteristics (Table 1) gives scanning electron microscopy (SEM), transmission electron microscopy (TEM) and atomic force microscopy (AFM) as techniques recommended for analysis. For this material, the elongated shape of the particles is the most restrictive property, with the consequence that techniques, which give as result an equivalent sphere diameter, are not recommended for its analysis.

\section{Conclusions}

A versatile technique-driven categorisation system for particulate materials is provided which takes into account the type of 
material (monotype or multiple types of particles, or article/ formulation), chemical composition, number of small dimensions and shape, expected size range, trade form and dispersibility, stability under testing conditions and other specific properties. The categorisation is linked to the performance profiles of commonly used particle sizing techniques. Using the proposed scheme considerably facilitates the selection of appropriate particle-sizing techniques with the objective to determine whether a material is a nanomaterial according to regulatory provisions. Based on measurements employing the most suitable techniques in terms of materials and regulatory purpose, decision criteria referring to the particle size or size distribution could be assessed in a reliable manner to be (i) fit for the purpose as well as (ii) accepted by different involved parties. Other criteria, if required for identification as nanomaterial in specific regulatory provisions, could be addressed in a similar way. The material categorisation can also serve as part of a knowledge base of a more elaborate decision system that also includes guidelines for specific measurement techniques, data evaluation and decision rules for identification, characterisation and quantification of nanomaterials. Moreover, it would help to implement the concept of nanomaterial within individual regulatory areas and facilitate its interpretation between different regulatory regions.

\section{Conflicts of interest}

There are no conflicts of interest to declare.

\section{Acknowledgements}

This work was funded by the European Community's Seventh Framework Programme (FP7/2007-2013) under Grant Agreement number 604347. The authors also would like to thank R. Kaegi (EAWAG, Switzerland), S. Wagner (University of Vienna, Austria), K. Löschner and E. H. Larsen (DTU, Denmark), T. Linsinger, R. Koeber and G. Roebben (JRC, Belgium), H. Marvin (RIKILT, The Netherlands), J. Tentschert (BfR, Germany) and P. Müller (BASF, Germany) for their helpful discussions and comments on the manuscript.

\section{References}

1 European Parliament and Council, Regulation (EU) 2017/745 of the European Parliament and of the Council of 5 April 2017 on medical devices, amending Directive 2001/83/EC, Regulation (EC) No. 178/2002 and Regulation (EC) No. 1223/2009 and repealing Council Directives 90/385/EEC and 93/42/EEC, Official Journal of the European Union, 2017, L 117/1, 1-175.

2 European Parliament and Council, Regulation (EU) No. 528/ 2012 of the European Parliament and of the Council of 22 May 2012 concerning the making available on the market and use of biocidal products, Official Journal of the European Union, 2012, L 167/1(1), 1-123.

3 European Parliament and Council, Regulation (EU) 2015/ 2283 of the European Parliament and of the Council of 25
November 2015 on novel foods, amending Regulation (EU) No. 1169/2011 of the European Parliament and of the Council and repealing Regulation (EC) No. 258/97 of the European Parliament and of the Council and Commission Regulation (EC) No. 1852/2001, Official Journal of the European Union, 2015, L 327, 1-22.

4 European Parliament and Council, Regulation (EC) No. 1223/2009 of the European Parliament and of the Council of 30 November 2009 on cosmetic products, Official Journal of the European Union, 2009, L342, 59-209.

5 U.S. Food and Drug Administration, Guidance for industry, Considering whether an FDA-regulated product involves the application of nanotechnology, Docket number: FDA2010-D-0530, 2014. http:/www.fda.gov/ RegulatoryInformation/Guidances/ucm257698.htm, accessed 10.08.2018.

6 U.S. Environmental Protection Agency, Chemical substances when manufactured or processed as nanoscale materials; TSCA reporting and recordkeeping requirements, Fed. Regist., 2017, 82, 3641-3655.

7 K. Rasmussen, B. Sokull-Klüttgen, I. J. Yu, J. Kanno, A. Hirose and M. R. Gwinn, in: Adverse Effects of Engineered Nanomaterials. Exposure, Toxicology, and Impact on Human Health, ed. B.Fadeel, A. Pietroiusti and A. A Shvedova, Elsevier, London, 2nd edn, 2017, Regulation and Legislation, pp. 160-189.

8 European Commission, Commission Recommendation 2011/696/EU of 18 October 2011 on the definition of nanomaterial, Official Journal of the European Union, 2011, L275/38, 38-40.

9 D. R. Boverhof, C. M.Bramante, J. H. Butala, S. F. Clancy, M. Lafranconi, J. West and S. C. Gordon, Regul. Toxicol. Pharmacol., 2015, 73, 137-150.

10 ISO 26824:2013, 1.1, Particle characterization of particulate systems - Vocabulary, 2013.

11 T. Linsinger, G. Roebben, D. Gilliland, D. L. Calzolai, F. Rossi, N. Gibson and C. Klein, Requirements on measurements for the implementation of the European Commission definition of the term "nanomaterial", $J R C$ Reference Report, EUR 25404 EN, Publication office of the European Union, Luxemburg, 2012.

12 K. Tiede, A. B. A. Boxall, S. P. Tear, J. Lewis, H. David and M. Hassellöv, Food Addit. Contam., Part A, 2008, 25(7), 795821.

13 G. Singh, C. Stephan, P. Westerhoff, D. Carlander and T. V. Duncan, Compr. Rev. Food Sci. Food Saf., 2014, 13, 693-704.

14 K. E. Sapsford, K. M. Tyner, B. J. Dair, J. R. Deschamps and I. L. Medintz, Anal. Chem., 2011, 83, 4453-4488.

15 F. Part, G. Zecha, T. Causon, E.-K. Sinner and M. HuberHumer, Waste Manag., 2015, 43, 407-420.

$16 \mathrm{~K}$. W. Powers, M. Palazuelos, B. M. Moudgil and S. M. Roberts, Nanotoxicology, 2007, 1, 42-51.

17 C. Szakal, S. M. Roberts, P. Westerhoff, A. Bartolomaeus, N. Buck, I. Illuminato, R. Candy and M. Rogers, ACS Nano, 2014, 8, 3128-3135. 
18 W. Wohlleben, J. Mielke, A. Bianchin, A. Ghanem, H. Freiberger, H. Rauscher, M. Gemeinert and V.-D. Hodoroaba, J. Nanopart. Res., 2017, 19, 61.

19 H. Godwin, C. Nameth, D. Avery, L. L. Bergeson, D. Bernard, E. Beryt, W. Boyes, S. Brown, A. J. Clippinger, Y. Cohen, M. Doa, C. O. Hendren, P. Holden, K. Houck, B. K. Kane, F. Klaessig, T. Kodas, R. Landsiedel, I. Lynch, T. Malloy, M. B. Miller, J. Muller, G. Oberdörster, E. J. Petersen, R. C. Pleus, P. Sayre, V. Stone, K. M. Sullivan, J. Tentschert, P. Wallis and A. E. Nel, ACS Nano, 2015, 9, 3409-3417.

20 C. Szakal, L. Tsytsikova, D. Carlander and T. V. Duncan, Compr. Rev. Food Sci. Food Saf., 2014, 13, 669-678.

21 CODATA-VAMAS, Uniform Description System for Materials on the Nanoscale Version 2.0 Prepared by the CODATA-VAMAS, Working Group on the Description of Nanomaterials, Version 2.0, 2016, DOI: 10.5281/zenodo56720.

22 A. G. Oomen, P. M. J. Bos, T. F. Fernandes, K. Hund-Rinke, D. Boraschi, H. J. Byrne, K. Aschberger, S. Gottardo, F. von der Kammer, D. Kühnel, D. Hristozov, A. Marcomini, L. Migliore, J. Scott-Fordsmand, P. Wick and R. Landsiedel, Nanotoxicology, 2014, 8, 334-348.

23 E. D. Kuempel, V. Castranova, C. L. Geraci and P. A. Schulte, J. Nanopart. Res., 2012, 14, 1029.

24 L. Y. T. Chou, K. Ming and W. C. W. Chan, Chem. Soc. Rev., 2011, 40, 233-245.

25 T. Anzai, M. Kaminishi, A. Porth, J. Handley and T. Satoh, Jap. J. Environ. Toxicol., 2012, 15, 17-29.

26 OECD, List of manufactured nanomaterials and list of endpoints for phase one testing of the sponsorshipprogramme for the testing of manufactured nanomaterials: revision, OECD Series on the Safety of Manufactured Nanomaterials, No. 27, ENV/JM/MONO, 2010, 46.

27 J. H. Arts, M. Hadi, M. A. Irfan, A. M. Keene, R. Kreiling, D. Lyon, M. Maier, K. Michel, T. Petry, U. G. Sauer, D. Warheit, K. Wiench, W. Wohlleben and R. Landsiedel, Regul. Toxicol. Pharmacol., 2015, 71, S1-S27.

28 Cambridge Dictionary: English, Cambridge University Press, 2018, https://dictionary.cambridge.org/, accessed 10.08.2018.

29 European Chemicals Agency (ECHA), Appendix 4: Recommendations for Nanomaterials Applicable to the Guidance on Registration Draft (Public), Version 1.0, 2016.

30 ISO 3252:1999(en), Powder metallurgy — Vocabulary, see definition 1115 'composite powder', 1999.

31 European Chemicals Agency (ECHA), Guidance on Requirements for Substances in Articles, Version 4.0, 2017.

32 European Committee for Standardization (CEN), Consumer products Definition, https:/www.cen.eu/work/areas/ consumerproducts/Pages/default.aspx, accessed 10.08.2018.

33 V.-D. Hodoroaba and J. Mielke, Techniques evaluation report for selection of characterisation methods, NanoDefine technical report, http://nanodefine.eu/ publications/reports/
NanoDefine_TechnicalReport_D3.1.pdf, accessed 10.08.2018

34 S. Wilbur, M. Yamanaka and S. Sannac, Characterization of nanoparticles in aqueous samples by ICP-MS., https:// www.agilent.com/cs/library/whitepaper/public/5991-

5516EN_WhitePaper_ICP-MS_NPs.pdf, accessed 10.08.2018.

35 F. Babick, J. Mielke, W. Wohlleben, S. Weigel and V.-D. Hodoroaba, J. Nanopart. Res., 2016, 18, 158.

36 S. C. Brown, V. Boyko, G. Meyers, M. Voetz and W. Wohlleben, Environ. Health Perspect., 2013, 121, 12821291.

37 N. B. Hartmann, K. A. Jensen, A. Baun, K. Rasmussen, H. Rauscher, R. Tantra, D. Cupi, D. Gilliland, F. Pianella and J. Riego Sintes, J. Toxicol. Environ. Health, Part B, 2015, 18, 299-326.

38 G. H. Michler, Problems Associated with the Electron Microscopy of Polymers in Electron Microscopy of Polymers, Springer Berlin Heidelberg, Berlin, Heidelberg, 2008, pp. 175-183.

39 D. B. Carlson and J. E. Evans, Low-Dose Imaging Techniques for Transmission Electron Microscopy, in The Transmission Electron Microscope, ed. K. Maaz, InTech, Rijeka, 2012, pp. 85-98.

40 R. F. Egerton, P. Li and M. Malac, Micron, 2004, 35, 399-409. 41 Y. Fujita, M. Mie and E. Kobatake, Biomaterials, 2009, 30, 3450-3457.

42 K. H. Kim, Z. Akase, T. Suzuki and D. Shindo, Mater. Trans., 2010, 51, 1080-1083.

$43 \mathrm{~J}$. Xie, Charging Mitigation Strategies in Imaging Insulating Polymer Spheres via Low Voltage Field Emission Scanning Electron Microscopy, Keysight Technologies, USA, 59912414EN, 2015, http://iterature.cdn.keysight.com/litweb/ pdf/5991-2414EN.pdf, accessed 10/08/2018.

44 A. De Lozanne, Microsc. Res. Tech., 2006, 69, 550-562.

45 M. S. Dresselhaus, G. Dresselhaus, R. Saito and A. Jorio, Phys. Rep., 2005, 409, 47-99.

46 C. Noguez, J. Phys. Chem. C, 2007, 111, 3806-3819.

47 The NanoDefine Methods Manual, ed. A. Mech and $\mathrm{H}$. Rauscher, JRC Technical Report (based on NanoDefine deliverables), in preparation, 2018.

48 R. Brüngel, J. Rückert, W. Wohlleben, F. Babick, A. Ghanem, C. Gaillard, A. Mech, H. Rauscher, S. Weigel, C. M. Friedrich, The NanoDefiner e -tool - A decision support framework for recommendation of suitable measurement techniques for the assessment of potential nanomaterials, in 2017 IEEE 12th Nanotechnology Materials and Devices Conference (NMDC), Singapore, 2017, pp. 71-72, DOI: 10.1109/ NMDC.2017.8350509.

49 OECD, Guidance on sample preparation and dosimetry for the safety testing of manufactured nanomaterials, Series on the Safety of Manufactured Nanomaterials No. 36, ENV/JM/ MONO(2012)40, 2012. 